\title{
Rhythm control strategies were not better than rate control strategies for atrial fibrillation
} Wyse DG, Waldo AL, DiMarco JP, et al. A comparison of rate control and rhythm control in patients with atrial fibrillation.
N Engl J Med 2002;347:1825-33.

\author{
QUESTION: Is a long term rate control strategy as effective as a rhythm control \\ strategy for atrial fibrillation $(\mathrm{AF})$ ?
}

\section{Design}

Randomised \{allocation concealed*\}†, blinded \{outcome assessors and monitoring committee $\}+, *$ controlled trial with a mean follow up of 3.5 years (Atrial Fibrillation Follow up Investigation of Rhythm Management [AFFIRM] study).

\section{Setting \\ 213 clinical sites in North America.}

\section{Patients}

4060 patients who were $\geq 65$ years of age (mean age 70 y, $61 \%$ men) or had other risk factors for stroke or death; had AF that was likely to be recurrent, likely to cause illness or death, and warranted long term treatment; and had no contraindications to anticoagulants. Follow up was $98 \%$.

Source of funding: National Heart, Lung and Blood Institute.

For correspondence: AFFIRM Clinical

Trial Center, Axio

Research, Seattle, WA,

USA.

Abstract and

commentary also

appear in ACP

Journal Club.

\section{Intervention}

2027 patients were allocated to rate control using the following drugs alone or in combination as selected by the treating physician: $\beta$ blockers, calcium channel blockers (verapamil and diltiazem), or digoxin. Target heart rate was $\leq 80$ beats $/ \mathrm{min}$ at rest and $\leq 110$ beats/minute during the 6 minute walk test. Continuous anticoagulation was required. 2033 patients were allocated to rhythm control using the following antiarrhythmic drugs alone or in combination: amiodarone, disopyramide, flecainide, moricizine, procainamide, leong@axioresearch.com

propafenone, quinidine, sotalol, or dofetilide. Cardioversion could be used if necessary. Continuous anticoagulation was encouraged, but could be stopped if sinus rhythm was maintained for $\geq 4$, but preferably 12 , consecutive weeks with antiarrhythmic drugs.

After failure of $\geq 2$ trials of either a rate control or rhythm control drug, patients could be considered for non-pharmacological therapy, such as radio frequency ablation, a maze procedure, and pacing techniques as appropriate to the randomised strategy. The goal for anticoagulation with warfarin was an international normalised ratio of 2.0-3.0.

\section{Main outcome measures}

The main outcome was overall mortality. A secondary outcome was a composite of death, disabling stroke, disabling anoxic encephalopathy, major bleeding, and cardiac arrest.

Rate control $v$ rhythm control for atrial fibrillation

\begin{tabular}{cllll} 
Outcome & Rate control & Rhythm control & RRI $(95 \% \mathrm{Cl})$ & NNT \\
\hline Mortality & $25.9 \%$ & $26.7 \%$ & $12 \%(-0.9$ to 28$)$ & Not significant \\
\hline
\end{tabular}

†Abbreviations defined in glossary; RRI, NNT, and Cl calculated from control event rate and hazard ratio reported in original article.

\section{COMMENTARY}

The AFFIRM trial and the RACE trial, along with 2 other recent randomised controlled trials-Strategies of Treatment in Atrial Fibrillation ${ }^{1}$ and Pharmacologic Intervention in Atrial Fibrillation²-support the current equivalence of rate control and rhythm control in most patients with AF. None of the trials found significant differences in variously measured endpoints, such as total mortality, cardiovascular related deaths, thromboembolic events, bleeding episodes, symptoms, and quality of life. These trials reflect the general demographics of patients with persistent or likely recurrent AF, with mean ages of $60-70$ years and high proportions of concomitant coronary heart disease, heart failure, and hypertension.

On the basis of these results, rate control should be the first therapeutic choice for many AF patients. Rhythm control is associated with high failure rates for maintaining sinus rhythm after cardioversion, a trend toward higher hospital admission rates (presumably because of the cardioversion procedures themselves), and a higher likelihood of drug toxicity and other adverse events. Pharmacological or electrical cardioversion, surgery, catheter ablation, pacing, and internal cardioversion devices are alternatives for patients in whom rate cannot be controlled. For younger patients with a first episode of $\mathrm{AF}$ and those who initially choose a "curative" approach, first line treatment using rhythm control is a reasonable alternative.

An additional advantage to rate control is the understood need to use aspirin, or more typically warfarin, indefinitely to prevent thromboembolic events. The AFFIRM and RACE trials allowed clinicians to stop antithrombotic therapy in rhythm controlled patients if they so desired, but most patients continued receiving antithrombotic preventive therapy. Guidelines support discontinuation of antithrombotic therapy in rhythm controlled patients after a period of stability. This, however, seems imprudent because rhythm is assessed infrequently in day to day clinical practice, AF recurrence is probable for most patients, and data show that patients with $\mathrm{AF}$ are more likely to have embolic events as a result of thrombi from other sources. ${ }^{4-6}$. 


\title{
Rate control was not inferior to rhythm control for recurrent persistent atrial fibrillation
}

\author{
Van Gelder IC, Hagens VE, Bosker HA, et al. A comparison of rate control and rhythm control in patients with recurrent \\ persistent atrial fibrillation. $N$ Engl J Med 2002;347:1834-40.
}

QUESTION: Is rate control inferior to rhythm control for persistent atrial fibrillation (AF)?

\section{Design}

Randomised \{allocation concealed*\}†, blinded \{outcome assessors and monitoring committee $\}$ †,* controlled, non-inferiority trial with mean follow up of 2.3 years (Rate Control vs Electrical Cardioversion for Persistent Atrial Fibrillation [RACE] Study).

\section{Setting}

31 centres in the Netherlands.

\section{Patients}

522 patients (mean age $68 \mathrm{y}, 63 \%$ men) with recurrent persistent $\mathrm{AF}$ or flutter, 1-2 electrical cardioversions during the previous 2 years, and no contraindications to oral anticoagulation. Exclusion criteria were arrhythmia lasting $>1$ year, New York Heart Association class IV heart failure, current or previous treatment with amiodarone, or a pacemaker. All patients were included in the analysis.

\section{Intervention}

256 patients were allocated to rate control, which comprised digitalis, a non-dihydropyridine calcium channel blocker, and a $\beta$ blocker, alone or in combination. Target resting heart rate was $<100$ beats/minute. 266 patients were allocated to rhythm control and had electrical cardioversion without previous treatment with antiarrhythmic drugs, after which they received sotalol, 160-320 mg/day. If AF recurred, electrical cardioversion was repeated, and sotalol was replaced by flecainide, propafenone, or amiodarone. Patients received acenocoumarol or fenprocoumon for electrical cardioversion. Oral anticoagulation could be stopped or changed to aspirin, $80-100 \mathrm{mg} /$ day, if sinus rhythm was present at 1 month.

\section{Main outcome measure}

A composite endpoint of death from cardiovascular causes, heart failure, thromboembolic complications, bleeding, need for pacemaker implantation, or severe adverse effects of antiarrhythmic drugs. Criterion for non-inferiority was an upper boundary of the $90 \%$ confidence interval $(\mathrm{CI}) \leq 10 \%$ for the difference between the incidence of the primary endpoint in the rate control group and the rhythm control group.

\section{Main results}

Analysis was by intention to treat. The rate control group was not inferior to the rhythm control group for the primary endpoint (table) or for the individual components of death from cardiovascular causes, heart failure, thromboembolic complications, bleeding, or pacemaker implantation. The rate control group had fewer severe adverse effects of antiarrhythmic drugs (table).

\section{Conclusion}

Rate control was not inferior to rhythm control for persistent recurrent atrial fibrillation and was associated with fewer severe adverse effects from antiarrhythmic drugs.

\section{*See glossary.}

$\dagger$ Information provided by author.
Sources of funding: Center for Health Care Insurance;

Interuniversity Cardiology Institute; 3M Pharma.

For correspondence: Dr IC Van Gelder, University Hospital, Groningen, The Netherlands. i.c.van.gelder@ thorax.azg.nl

Abstract and commentary also appear in $A C P$ Journal Club.
Rate control $v$ rhythm control for recurrent persistent atrial fibrillation at mean 2.3 years follow up

\begin{tabular}{lccl} 
Outcomes & Rate control & $\begin{array}{l}\text { Rhythm } \\
\text { control }\end{array}$ & $\begin{array}{l}\text { Absolute difference } \\
(90 \% \mathrm{CI})\end{array}$ \\
\hline Composite endpoint & $17.2 \%$ & $22.6 \%$ & $-5.4 \%(-11.0$ to 0.4$)$ \\
\hline Severe adverse effects & $0.8 \%$ & $4.5 \%$ & $-3.7 \%(-6.0$ to -1.4$)$ \\
\hline
\end{tabular}

†Abbreviations defined in glossary; composite endpoint = death from cardiovascular causes, heart failure, thromboembolic complications, bleeding, need for pacemaker implantation, or severe adverse effects of antiarrhythmic drugs. Criterion for non-inferiority was a $\mathrm{Cl}$ upper boundary $\leq 10 \%$.

\section{COMMENTARY — continued from previous page}

Given that rate control is currently a mainstay of AF treatment, is there a "best drug" for rate control? Probably not. But because cardiac disease and hypertension are common in patients with $\mathrm{AF}, \beta$ blockers such as metoprolol would be an appropriate first choice for patients who can tolerate this class of drugs. $^{7}$ The literature suggests that patients may require more than one drug for good rate control. ${ }^{3}$

Alan Silver, MD, MPH North Shore-Long Island Jewish Health System Lake Success, New York, USA

1 Carlsson J, Miketic S, Windeler J, et al. Randomized trial of rate-control versus rhythm-control in persistent atrial fibrillation: the Strategies of Treatment of Atrial Fibrillation (STAF) study. J Am Coll Cardiol 2003;41:1690-6.

Hohnloser SH, Kuck KH, Lilienthal J. Rhythm or rate control in atrial fibrillation-Pharmacological Intervention in Atrial Fibrillation (PIAF): a randomised trial. Lancet 2000;356:1789-94.

3 Fuster V, Rydén LE, Asinger RW, et al. ACC/AHA/ESC guidelines for the management of patients with atrial fibrillation: Executive summary. A report of the American College of Cardiology/American Heart Association Task Force on Practice Guidelines and the European Society of Cardiology Committee for Practice Guidelines and Policy Conferences (Committee to Develop Guidelines for the Management of Patients with Atrial Fibrillation) developed in collaboration with the North American Society of Pacing and Electrophysiology. Circulation 2001;104:2118-50.

Wyse DG. Rhythm management in atrial fibrillation: less is more [editorial]. J Am Coll Cardiol 2003;41:1703-6.

Manning WJ. Atrial fibrillation-rate versus rhythm control [letter]. N Engl J Med 2003;348:1284-6.

Falk RH. Management of atrial fibrillation-radical reform or modest modification? [editorial]. N Engl J Med 2002;347:1883-4.

Kühlkamp V, Seipel L. Atrial fibrillation-rate versus rhythm control [letter]. N Engl J Med 2003;348:1284-6. 